\title{
A Generic Phased Array Radar Model for Detailed Radar Performance Assessment
}

\author{
$\underline{\text { P.E. Berry }}^{a}$, G. Currie ${ }^{a}$ and D. Yau ${ }^{a}$ \\ ${ }^{a}$ Electronic Warfare \& Radar Division, Defence Science \& Technology Organisation, Edinburgh, South \\ Australia 5111 \\ Email:paul.berry@dsto.defence.gov.au
}

\begin{abstract}
The Defence Science and Technology Organisation of Australia undertakes the role of evaluating proposals for the acquisition and upgrade of military systems. Simulation is increasingly employed as a means of assessing military effectiveness and systems performance. This might be because the systems concerned exist only on paper or because there are too many variables affecting the system's performance that experimental trials would be prohibitively expensive on their own. In the domain of microwave radar, the Electronic Warfare \& Radar Division of DSTO has developed a detailed simulation model called GPARM (Generic Phased Array Radar Model) which represents the radio-frequency environment, the radar's antenna, and the radar's array and signal processing. The radio-frequency environment module of GPARM, in particular, is very detailed and represents the returns of radar signals from land and sea clutter, targets, emitters and the modulation of signals due to anomalous propagation effects.
\end{abstract}

The model is fully three-dimensional in order to capture the effects of clutter in the antenna sidelobes. This is particularly important for modelling modern digital phased array antennas which employ adaptive beamforming on receive to minimise the effects of spatially-distributed clutter and to spatially cancel deliberate jamming, because the receive weights used are a function of the signals returned from the three dimensional radio-frequency environment. Conventional two-dimensional models which represent a vertical slice through the main beam are unable to capture this aspect of a radar's performance. The simulation model is designed to be modular so that the domain expert in a particular aspect of the system and environment model, such as statistical clutter modelling or array signal processing, can contribute or modify a module of interest without having to comprehend every other aspect of the model's operation. These modules could collectively be regarded as populating a toolbox but as GPARM is a time-based simulation there needs to be an overall infrastructure to manage the time-stepping.

Whereas the most commonly-used radar system performance models, which are said to be parametric in the sense that they represent the effect of physical and algorithmic processes on the target signal to noise or clutter mean power ratio, GPARM works directly with the simulated time-sampled signals which originate from sampled probability density functions representing clutter and noise. Because of this, GPARM can explicitly process the sampled signals using the same signal and array processing algorithms which would be used in an operational radar, rather than use the approximate models for these processes used by parametric models, which usually make assumptions of Gaussian statistics. However, since the signals represent statistical samples of random processes, average performance has to be estimated using Monte Carlo simulation. Rather than explicitly represent the transmitted waveform at its carrier frequency, the effect of the attenuation of the signal by the environment in terms of amplitude and phase is represented at base-band, that is, as if the carrier had been down-converted to $0 \mathrm{~Hz}$. This enables the signals to be represented at the sample rate of the radar.

The purposes to which this model may be put are several-fold. It may be used to assess the performance of a radar system within a complex operational scenario which would be difficult to arrange in practice; to provide simulated data for the development and testing of signal processing, detection and tracking algorithms; for the off-line processing of measured data; and as a design tool for exploring trade-offs in the design of the overall system.

Keywords: Radar Analysis, Phased Array Antennas, Signal Processing, RF Propagation 


\section{INTRODUCTION}

A requirement exists within the Australian Department of Defence for a capability to assess the operational performance of radar systems, whether existing or proposed, by means of computer modelling and simulation. This requirement exists for a variety of reasons: a radar system of interest may not exist or may be in the design or developmental stage; the cost of testing a radar system for all possible sets of conditions under which it will be utilized operationally could be prohibitive; and a need exists to support in-house research through provision of simulated signal data for the development and evaluation of signal processing algorithms. Furthermore, a scalable approach to model fidelity is often required, such as for tender evaluation when insufficient information is available about proposed systems for high fidelity simulation, but sufficient for low fidelity exploration of their relative capabilities within a specified parameter space. This approach is also relevant to the needs of distributed military simulations running in real-time with humans in the loop, whether for training, tactical development or optimising effectiveness in the utilisation of new technologies. In this case a radar model may need to be simplified and to be to be executed efficiently, but still retain sufficient realism.

Computer-based radar system models have been in widespread use by radar manufacturers for many years. These are used to predict signal-to-noise ratio gains and losses in the propagation environment and radar hardware, and through the various stages of signal processing for the purpose of predicting detection and tracking performance at the design stage. These models tend to be either general-purpose spreadsheets and are very broad-brush, or are very detailed models of particular radar systems against which they have been carefully validated by the manufacturer over a long period of time. Such detailed system-specific models cannot easily be extended to other types of radar systems. The Commonwealth, as a purchaser and owner of many types of radars, frequently does not have access to these radar models or, if it does, cannot vouch independently for their correctness. As an informed purchaser of radar equipment the Commonwealth has therefore developed an independent capability to assess the performance of a range of radar system types based upon information provided by suppliers. This capability is used to provide informed decisions on acquisition and upgrade to the Department of Defence. An example of a naval radar model used by Canada's DRDC is described in Thomson (2002).

The high level requirements of this modelling and simulation capability are that the models should be sufficiently general to be able to analyse a range of radar systems in a variety of radio-frequency environments, should be sufficiently flexible to be able to be adapted to new modelling and analysis requirements, and should be easily comprehensible to a radar specialists who needs to be able to interpret and validate the results without having to wade through complex software architectures and interpret opaque code. This latter requirement means that the user or developer need not require specialist programming skills beyond knowledge of a high level language.

The model is aimed at a user community which comprises radar engineers and scientists who, while computer literate and familiar with high-level computer languages, may not be familiar with low-level languages and the practice of software engineering. There needs to be sufficient flexibility for them to apply the model to be used in new ways and to extend it, but at the same time for it to be well-structured and modular with clearly-defined and understandable interfaces. These requirements argue against the use of a graphical specification style of user interface (such as SIMULINK) where the modules are predefined and can only be used in predetermined ways, and even a GUI can be restrictive in terms of the freedom with which it allows the model to be used. Therefore it was proposed to develop the model in a high-level language which is widely used, namely MATLAB, as this is able to support the open architecture philosophy which facilitates users delving as deeply into the model as they need to. However, the MATLAB version is regarded as both a specification and a working prototype from which higher performance compiled and parallelized versions may be derived.

This paper describes a particular modelling and simulation framework called the Generic Phased-Array Radar Model (GPARM) which has been developed over a number of years by DSTO. It consists of two main modules: the radio-frequency environment module and the radar system module. The former computes the signals returned to the elements of a phased array antenna from the transmitted signals due to reflection by objects (referred to as targets) and land or sea (clutter), as well as subjected to attenuation due to atmospheric refraction, multipath effects, precipitation and diffraction; the latter emulates the processing of the returned signals by the radar using the actual algorithms used by the system of interest for determining the presence of targets of interest. 
This paper can only skim the surface of the radar knowledge domain but the principles of radar system design and performance analysis may be found in textbooks such as Barton (2005), Mahafza (2000), Peebles (1998) and Levanon (1988). A recent book covering specifically radar signal processing is Richards (2005).

\section{GPARM MODEL SPECIFICATION}

\subsection{Overview}

A mathematical model is specified which represents the essential features of a radar system to a required level of fidelity. Sacrifices in model fidelity are made for the sake of computational speed and a balance is struck between these competing requirements. Unlike parametric-style radar models which estimate mean signal powers, time is explicitly represented in order to represent temporal signal variations arising from various effects such as target Doppler shift, statistical environmental effects and receiver noise.

A radar typically transmits a series of radio-frequency pulses, referred to as a waveform, each pulse having a fixed amplitude and hence power. Although the pulse amplitudes are fixed, the frequencies or phases are usually modulated in time. The inter-pulse periods provide the opportunity to receive the returned signals without the receiver being swamped by a transmission. Whereas the transmitted waveform would ideally be modelled as a continuous function of time at its frequency of transmission, the fact that modern radars with digital signal processing sample the returned signals at discrete points in time provides a convenient time-discretisation for a computational model. The signals in the environment are therefore represented at discrete instants of time, as dictated by the radar system's sample rate, even prior to sampling, and the attenuation of those signals by the environment is modelled in terms of their effect on these sampled and down-converted signals.

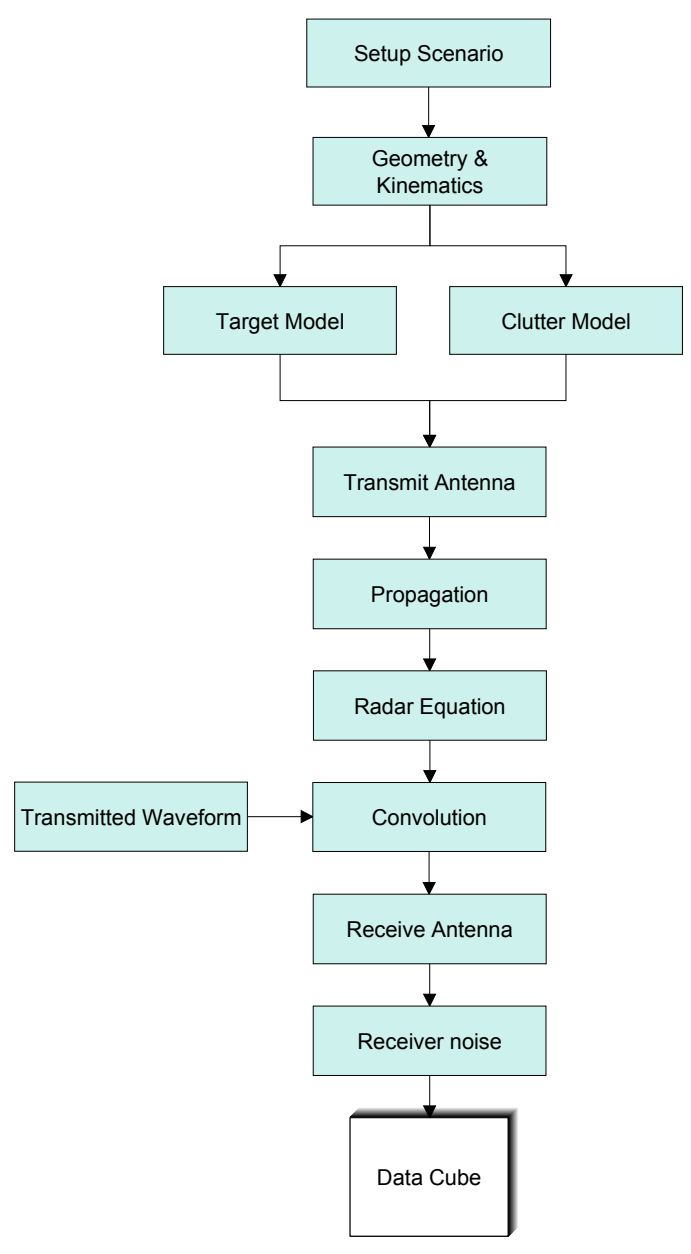

Figure 1. GPARM RF Environment Module Architecture

GPARM represents the geometry and kinematics of objects in three dimensional space and therefore has to perform a series coordinate transformations to obtain the coordinates of objects in the radar antenna's Frame of Reference. This is necessary because the strength of a signal received from an object at the antenna is dependant on the direction of the object relative to the antenna's orientation. Also, as the antenna may be on a moving platform this will need to be continuously updated during the course of a scenario.

\subsection{Physics}

The principal physical effects which are modelled in GPARM's radio-frequency environment module (see Figure 1) and which attenuate the discrete signals are: radar cross-section (RCS, the amount of power reflected from a target or clutter back to the radar based upon its geometry and orientation); Doppler effects due to relative motion between the radar and objects; propagation (specifically atmospheric refraction, diffraction around obstacles, multipath effects due to forward scatter reflection from surfaces causing signal cancellation, and scattering by rain). There may also be a need to represent other signal sources arising within the environment at the same or similar frequency, deliberate or otherwise, which could interfere with the radar's operation. In addition to these effects the signal powers are subject to an inverse-square law separately on transmit and receive based on the spatial separation between radar and object. This results in a signal power proportional to the inverse fourth power of the distance which is referred to as the Radar Equation. The consequence of this is that the received signal amplitudes are minute compared with the transmit signal amplitudes. These physical effects are clearly scenario-dependant but also frequencydependant. 


\subsection{Antenna}

The signals received at a phased array antenna at the sampling instants take into account the above physical effects but additionally model phase differences resulting from the fact that receive elements of the antenna have a slightly different spatial separation from the target based upon the configuration of the array and its orientation in space. These phase differences are significant compared with the wavelength of the radio waves and are critical for being able to electronically steer both the transmit and receive beams of a phased array antenna. The need for a detailed model of a phased array antenna has become important as phased arrays are increasingly being used for radar systems due to the convenience of electronic beamsteering over physically steered or rotating parabolic dish antennae. Another benefit is the fact that the individual transmit powers of the antenna elements can be relatively small, enabling solid-state devices to be used. This effect depends upon the configuration of the elements within the antenna which therefore has to be modelled. The antenna model is part of the RF Environment Module (Figure 1).

\subsection{Signal Processing}

The sampled signals are amplified and digitized by the radar hardware which can introduce further distortion due to nonlinearities that may need to be modelled. In particular the amplifiers introduce receiver noise which is usually modeled statistically as Gaussian noise. Following digitization of the sampled signals the subsequent processing in a modern radar is entirely computational and implemented in software based upon suitable algorithms. Typically the received signals are beamformed, pulse-compressed and Doppler filtered before being subject to a detection process. Each of these operations is described in the following. Beamforming is a linear spatial filter (i.e. weighted average) which maximizes the response due to signals originating from a particular direction. Therefore a target which is not in the specified direction will have a reduced response from the filter. Pulse compression is a linear filter which maximizes the signal to receiver noise ratio due to a target at a specified range. Doppler processing maximizes the signal arising from a target having a specified radial velocity by taking advantage of the fact that its returned signal is Doppler shifted. Since, in a surveillance mode, the radar system generally doesn't know where targets are to be found in angle or range nor know at what speed they may be traveling, all possible angles, ranges and velocities are searched in parallel in banks of filters. These are shown in the Signal Processing Module in Figure 2.

\subsection{Detection}

Finally, the presence of a target or otherwise is estimated by a detection algorithm which typically compares the signal arising from a particular angle and range, and with a particular radial speed, against a threshold. If a signal exceeds the threshold a detection is declared but if not then no detection is reported. This is the simplest possible detector but suffers from the problem that a signal may exceed the threshold due to extraneous effects such as clutter or receiver noise and therefore causing a false alarm, or a target signal may be too small to exceed the threshold. Of interest then is the probability of detection $P_{d}$ and probability of false alarm $P_{f a}$. A frequent requirement is to minimize the probability of false alarm by raising the threshold so as not to distract the radar operator unnecessarily, but this is always at the expense of reducing the probability of detection. More sophisticated detectors have been developed which adapt the threshold to maintain a constant false alarm rate, typically $P_{f a}=10^{-4}$ to $10^{-6}$, or which attempt to discriminate between noise, clutter and targets. Detection is also modelled in the Signal Processing Module of Figure 2.

Note that the signal processing and detection operations described above may be typical but in practice there may be considerable variations from these. In particular there are specialized modes for dealing with specific situations for example in an airborne radar when a target in the main beam is masked by clutter in a side-lobe which has the same radial velocity. In this case a space-time adaptive algorithm is used which is an adaptive filter applied simultaneously in space and slow-time (called a STAP processor). 


\subsection{Monte Carlo Simulation}

Ultimately a radar system's performance is assessed in terms of its detection probability. Since the signals originating from targets and clutter are statistical, that is they are sampled from probability distributions, and the end result is the declaration of a detection or not, the model needs to be run repeatedly as a Monte Carlo simulation and the results statistically processed to estimate the overall detection and false alarm probabilities (see Figure 3). For this reason the model needs to be as efficient as possible with the creation of compiled and parallelized versions to speed up execution times. This will also be the case if the model is to be run in real time, for example to represent a radar system in a distributed military simulation with a human-in-the-loop either for training or to develop improved tactics to exploit new technologies.

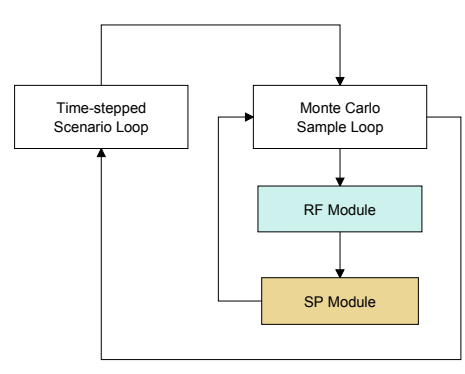

Figure 3. GPARM can be run within a Monte Carlo loop and/or a timestepping scenario loop.

\section{GPARM SOFTWARE ARCHITECTURE}

\subsection{Overview}

We have seen that the radar system model needs to be capable of being reconfigured easily to handle different scenarios and radio-frequencies, different antenna configurations, different radar system hardware and different signal processing schemes. This requirement impacts upon the software architectural design of the model which needs to be capable of being adapted readily to meet these various needs.

\subsection{Modular Decomposition}

The model has been decomposed as far as possible into self-contained modules so as to separate the representation of the independent physical effects which attenuate the signals received at the radar. This both facilitates understanding of the software system by breaking it down and enables domain experts to contribute stand-alone physical modules with clearlydefined interfaces without the need to understand the entire software system. The modules representing

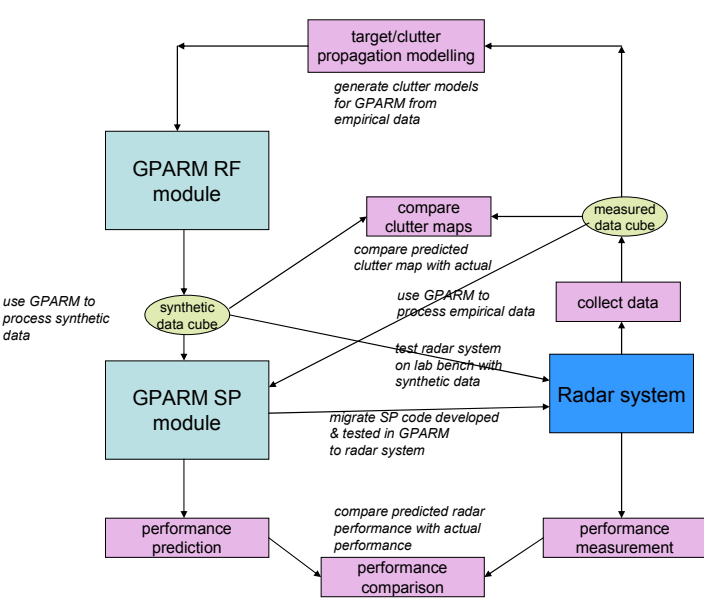

Figure 4. This diagram demonstrates the multiple uses to which GPARM may be put in either generating synthetic data or processing real data off-line.

physical effects can be written as generally as possible so as to accommodate any requirement through choice of parameter values, or be sufficiently self-contained that they can be interchanged with modules of varying fidelity and computational efficiency without impacting upon the rest of the software. A simplified flow chart is shown in Figure 1 which belies the true complexity of the model. In fact the propagation model represented by a box is itself a complex physical model and the box representing the convolution is the most computationally demanding part of the model.

The modules representing the signal processing, however, correspond to specific mathematical algorithms and are generally unique to a particular radar system, if not in terms of the algorithm then certainly in terms of parameter values. These are therefore collected into an ever-expanding library of functions which is extended as the model is applied to new radar systems. They are called by a radar system-specific module which processes the signal data in the correct sequence and determines the detection or otherwise of a target. It follows that GPARM is not a single model as such as its architecture needs to be modified to represent new radar systems. Therefore we describe it as a radar modelling system framework. The key to facilitating its application to many different types of radar is in the design of that framework.

It was seen from the previous section that we have contradictory requirements: an open software architecture for ease of comprehension and to facilitate contributions form a user community which does not consist of specialist programmers which argues for a high-level language, yet efficient execution which argues for a low-level language. 


\subsection{Code}

MATLAB was selected as a suitable high-level language in which to write the model due to its widespread use. As the model increased in complexity it became clear that software engineering techniques would need to be adopted to manage the software development, particularly when several people were working on it simultaneously. Version control was therefore instituted using CS-RCS developed by ComponentSoftware Inc. (http://www.componentsoftware.com/). Similarly, documentation was considered critical to understanding the model, both explaining the code and also explaining the physical models and their numerical implementation as well as the signal processing algorithms. A basic graphical user interface was provided but found to be constraining to the user who wished to exploit the full flexibility offered by GPARM.

\subsection{Execution Speed}

Where available, the GPU is used to optimize the computationally intensive functions such as the clutter return calculation. The clutter cells are arranged on a range/azimuth grid. The azimuth interval may typically be one degree or less. The range interval is determined by the system sample rate and may be tens of metres in length. This defines a large number of cells in range and azimuth. Specialised multi-threaded algorithms to compute the antenna gain to each clutter cell and the clutter cell signal azimuth sum for each range cell have been developed using CUDA (Compute Unified Device Architecture), a parallel computing architecture developed for NVIDIA GPU's.

\section{MODEL APPLICATION}

The ways in which GPARM may be utilized by radar analysts, designers and researchers in conjunction with measured data is several-fold, as shown in Figure 4:

- The RF environment module can be used to generate a synthetic data cube for subsequent analysis and signal processing research.

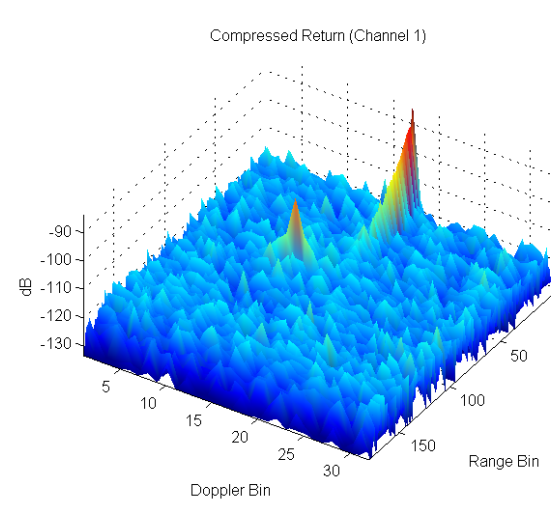

Figure 5. This diagram demonstrates the multiple uses to which GPARM may be put in either generating synthetic data or processing real data off-line.

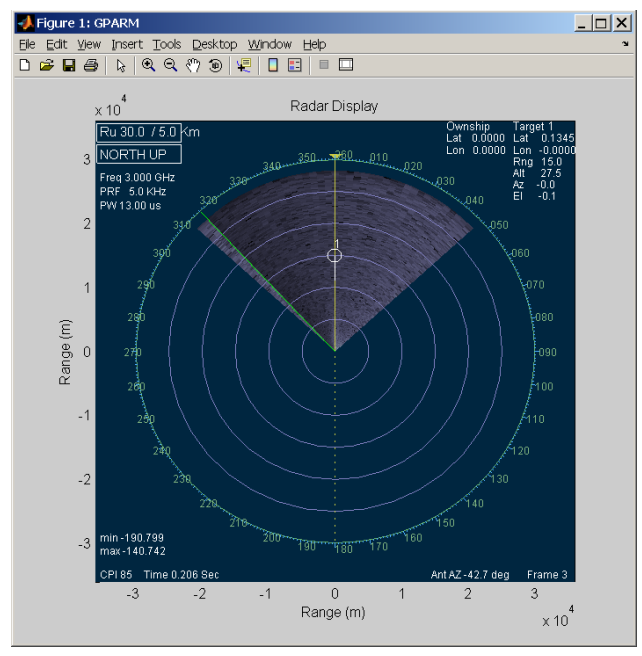

Figure 6. GPARM can be run in real time with detections shown on a radar display depending upon radar and scenario complexity.

- The signal processing module can be used to process a data cube off-line, whether real or synthetic, for subsequent radar system performance assessment and signal processing research.

- $\quad$ The clutter and propagation models used by GPARM can be validated against measured data by making a comparison between the predicted signals returned from the RF environment and the measured signals by comparing clutter maps.

- The signal processing module can be used to develop the signal processing algorithms and test the code prior to their migration to the radar system's processor.

- Synthetic data generated by GPARM can be used to stimulate a radar system on the lab bench prior to fielding.

- The radar system's predicted performance can be compared against its actual performance. Any difference would be investigated either to enhance the model or improve understanding of a radar system's capabilities.

A typical range-Doppler clutter map following beamforming, pulse compression and Doppler processing is shown in Figure 5 which shows a moving target which has been distinguished from stationary clutter. This is 
referred to as a clutter map. The application of a detection algorithm to this data is typically displayed to the radar operator as shown in Figure 6.

\section{DISCUSSION AND CONCLUSIONS}

GPARM is a radio-frequency environment and radar system model capable of simulating the signals returned from complex scenarios and processing them for the purposes of assessing radar system performance. It has been under development for several years and is now in the process of being validated and finding application in radar acquisition studies. The need to continuously adapt the model to new requirements has required particular care to be given to designing its software architecture to promote comprehension and reuse. Considerable improvements have been made in its speed of execution through the exploitation of multiprocessors and GPUs. It is planned to extend it to incorporate multi-target tracking and resource management.

\section{ACKNOWLEDGMENTS}

This paper has been approved for public release by Chief, Electronic Warfare \& Radar Division, DSTO. The authors would like to thank DSTO for permission to publish this work.

\section{REFERENCES}

Thomson, A.D. (2002) A Target Simulation for Studies of Radar Detection in Clutter. DRDC Canada, TR 2002-145.

Barton, D. K. (2005). Radar System Analysis and Modeling. Artech House.

Mahafza, B.R. (2000). Radar System Analysis and Design using MATLAB. Chapman \& Hall / CRC.

Peebles, P.Z. (1998). Radar Principles. Wiley Interscience.

Levanon, N. (1988). Radar Principles. Wiley.

Richards, M.A. (2005). Fundamentals of Radar Signal Processing. McGraw-Hill. 